\section{BRIGHTER ILLUMINATION TO TREATMENT AREA}

NSK's powerful surgical micromotor, Surgic XT Plus, features accuracy, speed, torque and control. The Surgic XT Plus brings a new dimension of control through the application of NSK's unique Advanced Torque Calibration (ATC). ATC enables the Surgic XT Plus to calibrate the micromotor to the rotational resistance of the individual handpiece, offering reassurance that you are working with the optimum torque and speed settings and so ensuring accurate, safe and smooth operations.

The Surgic XT Plus responds to operator commands instantaneously, giving you extra confidence when making the precise movements required to meet the demands of implant procedures.

Developed and designed with advice from implant dentists, the Surgic XT Plus also benefits from built in optic illumination. The application of cellular

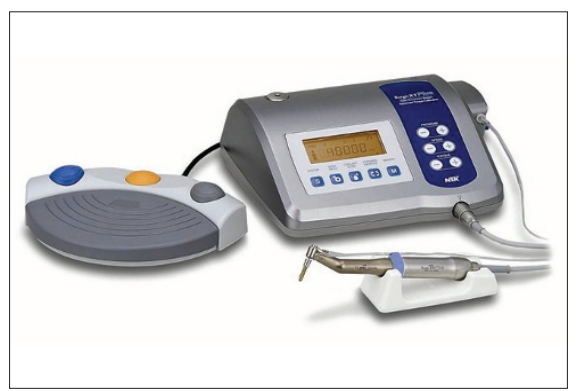

glass optics delivers 20\% brighter illumination to the treatment area, making complex and often prolonged implant procedures easier to perform.

Combine this with the new and improved LCD display, which offers excellent visibility and clearly shows speed and torque values simultaneously, and you have a completely user-friendly system that guarantees safety and accuracy in surgical procedures.

Reader response number 59

\title{
ANTI-INFLAMMATORY AND ANTIBIOTIC ELEMENTS
}

Ledermix Dental Paste, available from Blackwell Supplies, is simple to use, versatile and cost-effective and an ideal intracanal medicament. Its antiinflammatory and antibiotic elements assist in providing effective pain relief until definitive root canal therapy can be performed.

Ledermix is available in combination kits including the Dental Paste, the Dental Cement and Hardeners

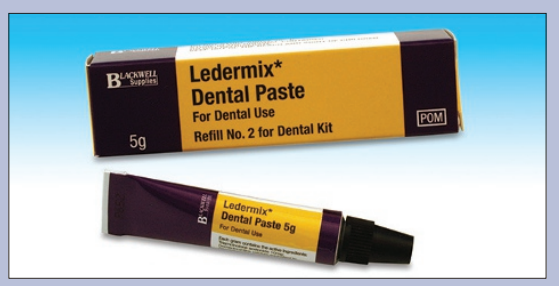

for fast or slow setting. The Cement can be used as a pulp-capping agent and as a temporary sublining for deep cavities where no exposure has occurred.

Ledermix combines the antibiotic properties of Demeclocycline and the anti-inflammatory properties of Triamcinolone for reliable pain relief during endodontic therapy.

Ledermix Dental Paste is also watersoluble, so it can be easily rinsed out of the treatment site in preparation for further treatment. Blackwell Supplies has also produced a free patient leaflet giving advice on oral care, available for all practices.

Reader response number 60

\section{LOW-VIBRATION}

SCALING DEVICE

The Symmetry IQ 4000 from HuFriedy is a new ultrasonic scaler. This low-vibration scaling device is highly efficient and at the same time ensures the highest levels of comfort for the user and the patient.

To achieve optimum results the user merely has to maintain a $90^{\circ}$ angle between the tip and the tooth. It is not necessary to exert any pressure. The ultrafine Symmetry perio tips deliver effective scaling performance in very deep pockets without causing pain to the patient.

In the interests of patient comfort the irrigating solution can be heated. The versatile dispenser bottle allows the clinician to deploy various irrigating solutions, independently of the mains water supply.

The Symmetry IQ 4000 has a lightweight, ergonomically shaped handpiece. The integrated fibre optic ensures that the treatment site is brightly illuminated, while the userfriendly touch pad with its power presets allows the user to select the various treatment modes at the touch of a button. The convenient memory function saves the settings between treatment sessions.

Reader response number $\mathbf{6 1}$

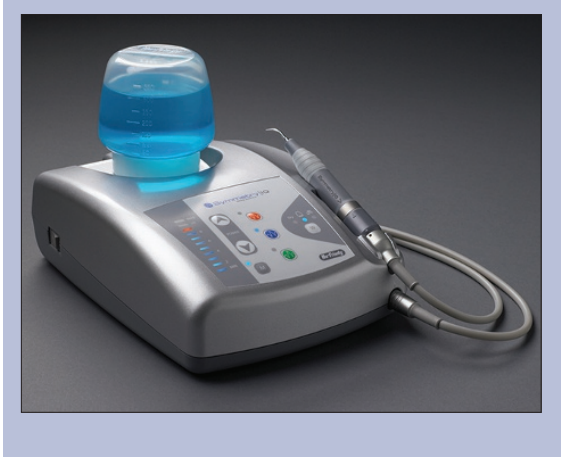

\section{ENSURE LONGEVITY OF THE FINAL RESTORATION}

New from Shofu is Beautifil II Gionomer. This composite restorative material is based on Pre-Reacted Glass Ionomer technology which combines the benefits of glass ionomers with those of composite resins to produce a biocompatible, fluoride releasing, high strength aesthetic material.
Due to the on-going release and recharge of the fluoride particles within Beautifil II, secondary caries is no longer a concern with the added benefit that plaque will not adhere to the surface of a Beautifil II restoration.

Saliva forms a material film on the surface of Beautifil II that is reported to minimise plaque adhesion and inhibit bacterial colonisation as an on-going process.

To claim your free Beautifil II sample (without obligation), which includes $3 \times$ single dose A2, $3 \times$ single dose A3 and instructions for use, please contact Shofu on 01892870800.

Reader response number 62 